\title{
EEG OBSERVATIONS IN DYSTROPHIA MYOTONICA (CURSCHMANN-STEINERT)
}

\author{
R.S.H.M. BEIJERSBERGEN, A. KEMP and W. STORM VAN LEEUWEN \\ Department of Clinical Neurophysiology, University Hospital, Nicolaas Beetsstraat 24, 3511 HG Utrecht (The \\ Netherlands)
}

(Accepted for publication: September 11, 1979)

In the past, various investigators have described electroencephalographic abnormalities in patients suffering from dystrophia myotonica (Savoldi 1959; Friedlander and Bittenbender 1964; Lee and Hughes 1964; Barwick et al. 1965; Lundervold 1969; Lundervold et al. 1969). Lundervold et al. (1969) studied 49 patients with this disease and observed EEG or pneumoencephalographic abnormalities in 43 of them. In a follow-up study of 17 of these patients they observed that in some of the cases the EEG abnormalities preceded anatomical changes. Of their entire group, 23 patients had obvious neurophysiological disturbances of the central nervous system.

In 1953 one of us (A.K.) became particularly interested in this disease, following the admission to this clinic of two siblings suffering from it. The EEGs of these patients, reported on by one of us (W.S.v.L.), were not specifically abnormal, but both of them showed some peculiarities. These consisted in a low frequency alpha rhythm of small amplitude, persisting while the eyes were open, and in a scarcity of other EEG phenomena. In keeping with the muscular dystrophy, the EEGs contained little or no muscle artefacts.

In the course of a subsequent intensive study (A.K.), involving most of the patients with dystrophia myotonica in The Netherlands, EEG examinations were carried out of these patients. Thus, over the period elapsed since this time a comparatively large number of EEGs recorded from patients suffering from this rare disease has been assembled.
The originally observed peculiarities were encountered again in some of these patients, but not, or not so clearly, in others. To investigate whether these peculiarities could be substantiated, one of us (R.B.) has reviewed the entire material anew.

In doing so, some other aspects were also considered. The aspects of alpha rhythms investigated were: (1) frequency, (2) amplitude, (3) indices, with the eyes closed as well as open (alpha rhythm persistency). In 8 cases it has been possible to determine by means of EEG computer analysis two other parameters of the alpha rhythms, i.e., their bandwidths and their bilateral and longitudinal coherences.

In addition to these aspects some other parameters were considered: (1) the occurrence of activities at low frequencies, (2) the presence of irritative phenomena: sharp waves, spikes and spikes and waves.

\section{Patients}

The group consisted of 84 patients, examined in the neurological clinic in the period between 1953-1976. Of these 59 were male and 25 female. The majority of the patients (76) were hospitalized for a short or longer period of time; 8 patients were seen only in the outpatient department. The ages of the patients when investigated varied between 14 and 60 years (mean 36, S.D. 12). The diagnosis was made on the basis of the relevant clinical criteria. In 9 patients 2 EEGs and in 2 
patients 3 EEGs were obtained per patient. Thus a total of 97 EEGs was available.

\section{Methods}

\section{Recording}

The early EEGs (42) were recorded on 6 channels; later ones were obtained with 8 - or 16-channel EEG apparatus (Van Gogh penwriters). The last 29 were recorded by means of 16-channel inkjet EEG apparatus (Elema Schönander); samples of the last 6 of these were stored on magnetic tape. These samples were subsequently submitted to computer analysis, for calculating frequency power spectra by means of Fast-Fourier analysis, and bilateral and longitudinal coherence functions (subsequently the EEGs of 2 new patients have been analysed).

\section{Measurements}

The frequency of the alpha rhythm was determined by manual measurement over 2 periods of $10 \mathrm{sec}$ in the parieto-occipital derivations. The first period was selected from the beginning of each recording session and the second from the end. The results were averaged. In 5 EEGs it was not possible to measure an alpha frequency because the traces were irregular or did not contain a sufficient quantity of alpha rhythm.

The alpha index was measured over a period of $30 \mathrm{sec}$ while the patient's eyes were open, and over another period of $30 \mathrm{sec}$ while the eyes were closed. (Alpha index is the percentage of time alpha waves with amplitudes above a given level, occur over a certain period of time.)

The amplitudes were obtained from the data presented in the original EEG reports. The values were obtained in the usual way by measuring alpha waves in various places. Such measurements tend to favour the largest amplitudes.

The degree of regularity or irregularity of the alpha rhythms was evaluated from the reports made at the time by different qualified electroencephalographers. The alpha rhythms had been classified as monotonous, very regular, regular, irregular or very irregular. Although these classifications were obviously subjective it should nevertheless be taken into account that it has been a custom in this department first to describe the EEGs and subsequently to evaluate them against the clinical data. The descriptions regular or irregular alpha rhythm have therefore been made without information concerning the patient's complaints. The number of EEGs from patients with dystrophia myotonica is small in relation to the total number of EEGs recorded over this period (approx. 60,000).

From the last 6 EEGs in which frequency power spectra were computed it has been possible to obtain more quantitative information concerning a) the value of the dominant alpha frequency, b) the width of the alpha frequency band (indicative of the degree of irregularity), and c) the degree of bilateral and longitudinal coherence (Storm van Leeuwen et al. 1975).

\section{Severity of the affliction}

The patients were grouped according to severity of the disease into 5 groups: (I) subjectively free of complaints, but some clinical symptoms of the disease present; (II) subjective complaints, still able to carry out normal work; (III) strong subjective complaints, with impediment of usual work; (IV) severe subjective complaints, severe clinical symptoms, no longer able to carry out usual work; (V) severe subjective complaints, severe clinical symptoms, complete helplessness.

\section{Results}

\section{(1) Classification}

The classification with respect to normality or degree of abnormality of the EEGs was as 
follows. (1) No abnormalities, 38 (45\%). In 5 of these, however, epileptiform phenomena were provoked by intermittent photic stimulation (IPS). (2) Low frequency alpha rhythms, at $8.0 \mathrm{c} / \mathrm{sec}$ or lower, were encountered in 26 EEGs (31\%). Of these, 4 were of low voltage. Too much diffuse theta activity occurred in 1 EEG. Focal theta activity was observed in one, focal epileptiform activity in one and an epileptiform reaction to IPS in 2 EEGs. (3) Too much diffuse theta activity was observed in 10 EEGs (12\%), 2 of which in addition had focal epileptiform phenomena. (4) In 5 cases (6\%) the EEGs were classified as of low voltage. (5) In 2 EEGs $(2 \%)$ the only abnormality was focal epileptiform activity. (6) In 3 EEGs (4\%) very irregular activity was encountered with alternately fast and slow diffuse activities and sporadic high frequency alpha rhythm. These 3 EEGs came from 3 siblings in an early phase of the disease.

From the above it appears that there were a comparatively large number of low voltage EEGs (9), of EEGs with focal epileptiform activity (5) and with epileptiform activity provoked by IPS.

\section{(2) Alpha frequency}

In 92 EEGs it was possible to measure comparatively precisely the frequency of the alpha rhythms (see Methods). The results are shown in Fig. 1. From this figure it appears that the alpha frequencies in many cases were low. The entire frequency histogram appears to be shifted to the low frequency end in comparison to normal (Friedlander and Bittenbender 1964). The mean of the alpha frequencies in all patients was $8.5 \mathrm{c} / \mathrm{sec}$ (S.D., 1.0 ; S.E., 0.1 ), whereas in a normal group of subjects it is $9.9 \mathrm{c} / \mathrm{sec}$ (S.D., 1.0; S.E., 0.1) (Wieneke et al. 1980).

\section{Alpha index}

Eyes closed. The alpha index with the eyes closed was high (mean, 77; S.D., 21; S.E., 2.2) in most cases (see Fig. 2). Since no data were available on alpha index in a com-

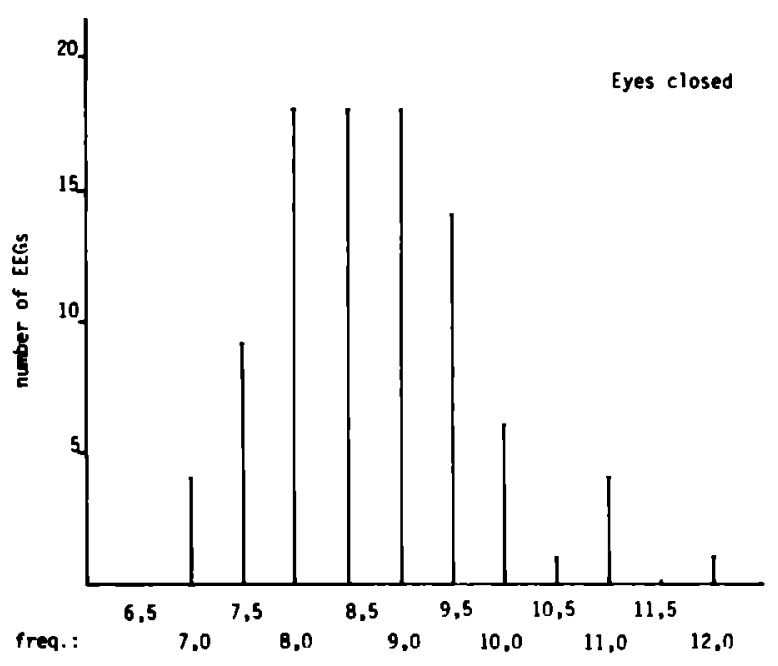

Fig. 1. Alpha frequencies encountered in 92 EEGs. Vertical axis: number of EEGs in which alpha frequency could be determined. Horizontal axis: dominant alpha frequencies.

parable group of normal subjects, no comparison with normals could be made. From Fig. 2 the relation between the alpha index and the alpha frequency can also be seen. From this

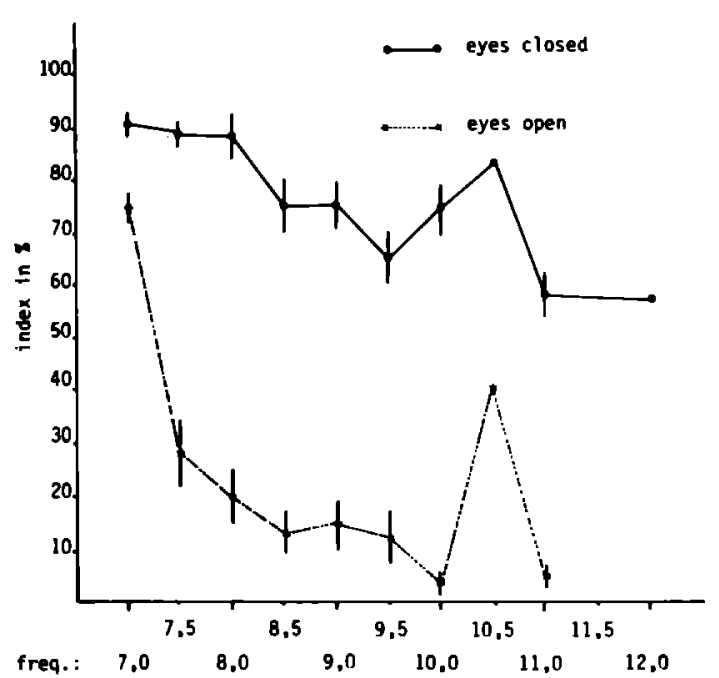

Fig. 2. Relation between alpha index and alpha frequency determined in 91 EEGs. Vertical axis: mean alpha index in percentages. The vertical bars indicate the standard errors. Horizontal axis: dominant alpha frequencies. $-\longrightarrow$, indicate the values obtained during eyes closed; $x$......-x during eyes open. Note a tendency for the indices to be highest at the lowest frequencies. 
diagram it appears that the lowest alpha frequencies tend to be related to the largest alpha indices and the highest alpha frequencies to relatively small alpha indices. With the eyes open this trend appears to be more pronounced than with them closed.

Eyes open. The alpha indices with the eyes open averaged over the entire group were considerable smaller than with the eyes closed (18; S.D., 24; S.E., 2.5). In this condition there were considerable individual differences. In 21 subjects the alpha index was 0 and in 19 subjects it was 40 or larger. Because of this, the variance was high (136\%). Nevertheless, in this condition it can also be seen that the largest indices were related to the lowest frequencies.

\section{(3) Amplitude of alpha rhythm}

The amplitudes of the alpha rhythms were small (see Fig. 3). The average amplitude over the entire group was $44 \mu \mathrm{V}$ (S.D., 14.4; S.E., 1.5). Comparison between alpha frequency and voltage did not demonstrate obvious relations (Fig. 3).

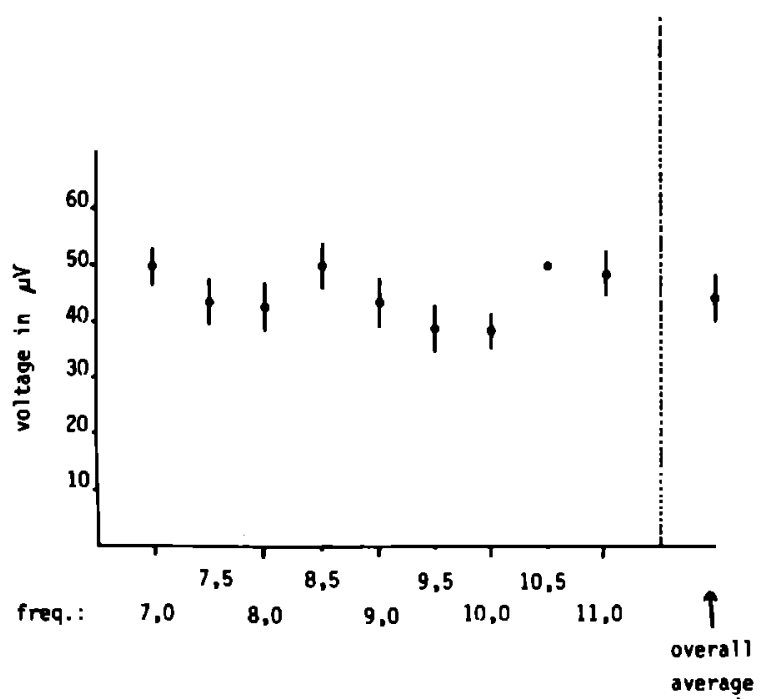

Fig. 3. Relation between alpha frequencies and amplitudes measured in 92 EEGs. Vertical axis: mean voltage in microvolts. Vertical bars indicate the standard errors. Horizontal axis: alpha frequencies.

\section{(4) Regularity of alpha rhythm}

In 89 EEGs the alpha rhythm was described in the reports made at the time as regular or monotonous. Out of the 24 EEGs with an alpha frequency of $8 \mathrm{c} / \mathrm{sec}$ or lower only one was described as irregular.

\section{(5) Computer analysis of EEGs}

Computer analysis has been performed of the EEGs of 6 of the patients. Subsequently it has been possible to perform computer analysis of the EEGs of 2 more patients suffering from the Curschmann-Steinert disease. In the following, analysis results will be described of EEGs recorded from 8 patients (2 of them therefore not included in the group of 84).

Power spectra were computed from 8 derivations, 4 at each side of the head. Analyses obtained from one of these patients are shown in Fig. 4. The method included the calculation of the values of the dominant peak frequencies (frequency selectivity $0.5 \mathrm{~Hz}$ ), their powers in decibels and their bandwidths (at half power level). These latter values can be used for quantifying the degree of regularity of rhythms. Because of the limited frequency selectivity a bandwidth of $0.6 \mathrm{~Hz}$ is obtained with a pure sine wave generator. Dominant peak frequencies with a bandwidth of $0.7-0.8 \mathrm{~Hz}$ were classified as very regular (5 patients), $0.9-1.0 \mathrm{~Hz}$ as regular (3 patients) and $1.0 \mathrm{~Hz}$ and broader as irregular (none of the patients). In Table I the bandwidth is split up into two parts: the difference between the lower limit and peak frequencies and the difference between the peak frequency and the upper limit. These two parts differed not at all or by only $0.1 \mathrm{~Hz}$ in most cases. The peaks were clearly symmetrical. In addition, coherences per frequency were calculated for homologous derivations on the two sides of the head, called bilateral coherences, and for the anterior and posterior parts of the head, called longitudinal coherences (for further details see Storm van Leeuwen et al. 1975). The analysis data obtained from the patients 

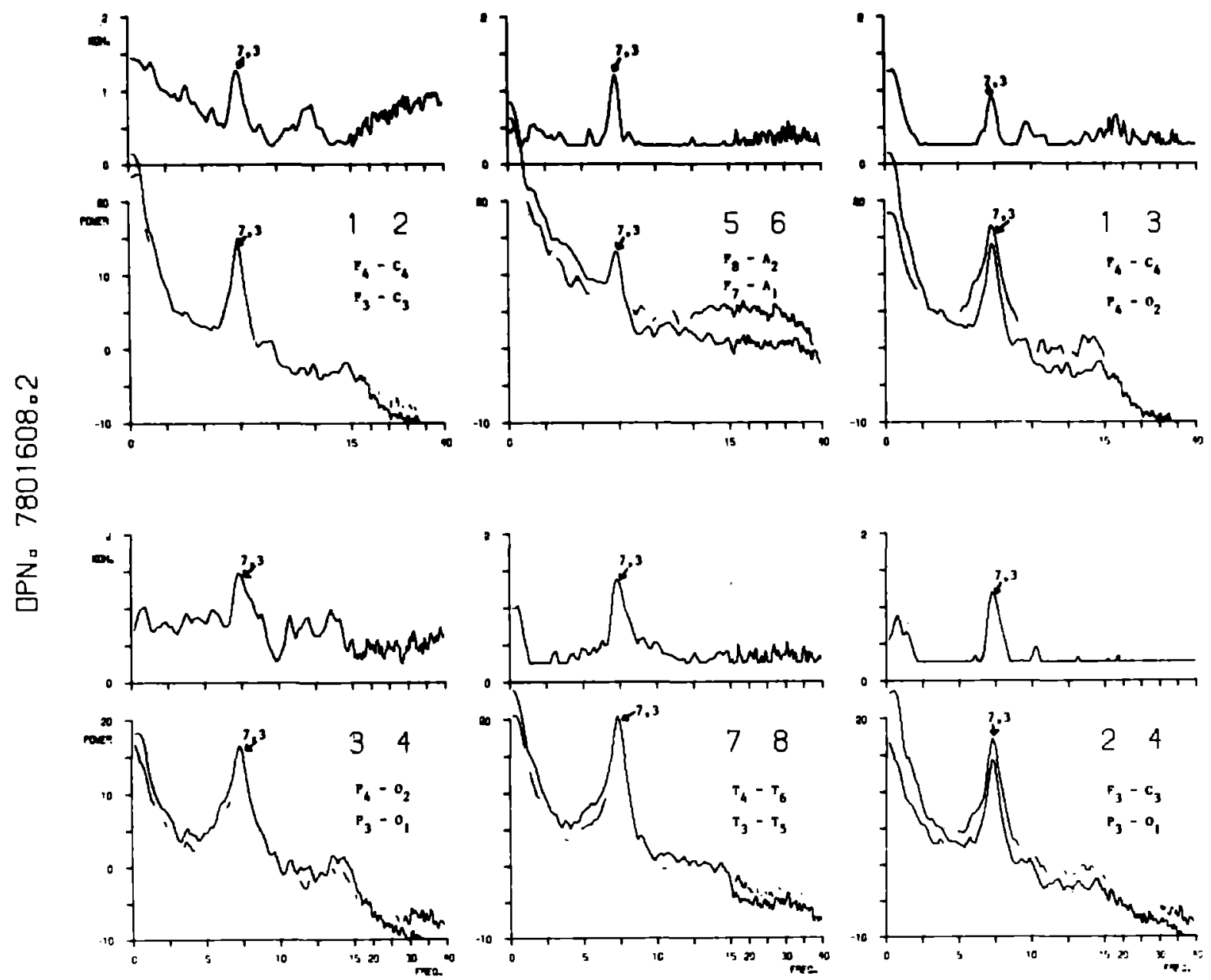

Fig. 4. Six pairs of power spectra calculated for one of the patients from 8 derivations, indicated by the inserts. The 4 pairs of analyses at the left of the figure concern comparison of derivations from homologous left-right areas. The 2 pairs of analyses at the right of the figure concern comparison of anterior and posterior derivations. The uninterrupted lines represent the spectra from the first of each pair of derivations. The spectra of the second derivation are drawn only where they differ significantly from the first. Above each pair of spectra the coherences (Koh) are presented. The values of power are presented logarithmically in decibels. Note low alpha frequency (7.3 c/sec) and high left-right and antero-posterior coherences.

are presented in Table I.

From this Table it appears that in most of these cases the alpha rhythm was regular or very regular, the frequencies were low or very low, the bandwidths small, the coherence values generally high and the power values intermediate. In this context it should be remarked that an alpha rhythm with a narrow bandwidth and with a large index may correspond to a large energy value even though the amplitude is comparatively small. Measured by hand in this small group the alpha amplitudes ranged between 20 and $60 \mu \mathrm{V}$.

\section{(6) Severity and duration of the affliction}

We have tried to relate the above parameters to (a) the severity and (b) the duration of the illness at the time of the EEG recording. The evaluation of both these aspects was diffi- 


\section{TABLE I}

Bandwidths of the alpha peaks are split up in two parts: below $(-)$ and above the peak frequency. EdB indicates the power in decibels of the alpha peak frequencies $\left(0 \mathrm{~dB}=0.6 \mu \mathrm{V}^{2} / \mathrm{Hz}\right)$. Bico and loco indicate the values of bilateral and longitudinal coherences. These values are expressed after a z-transformation (Jenkins and Watts 1968) (the value 2.0 correlates with approx. $95 \%$, the value 1.0 with approx. $40 \%$ coherence). $\bar{X}=$ mean of 8 patients; S.D. = standard deviation of group; S.E. = error in mean based on the standard deviation.

\begin{tabular}{|c|c|c|c|c|c|c|c|}
\hline $\begin{array}{l}\text { Patient } \\
\text { no. }\end{array}$ & $\begin{array}{l}\text { Alpha } \\
\text { freq. } \\
\text { (c/sec) }\end{array}$ & \multicolumn{2}{|c|}{$\begin{array}{l}\text { Bandwidth } \\
\text { below, above } \\
\text { peak frequency } \\
\text { (c/sec) }\end{array}$} & $\begin{array}{l}E d B \\
(d B)\end{array}$ & $\begin{array}{l}\text { Bico } \\
\text { (z-scale) }\end{array}$ & $\begin{array}{l}\text { Loco } \\
\text { (z-scale) }\end{array}$ & $\begin{array}{l}\text { Age } \\
\text { (years) }\end{array}$ \\
\hline $1251 / 75$ & 9.0 & -0.5 & 0.5 & 10 & 1.1 & 1.1 & 25 \\
\hline $2760 / 75$ & 8.4 & -0.4 & 0.5 & 17 & 1.9 & 0.7 & 20 \\
\hline $320 / 76$ & 7.9 & -0.4 & 0.4 & 20 & 2.0 & 1.0 & 29 \\
\hline $554 / 76$ & 7.2 & -0.3 & 0.4 & 18 & 2.2 & 0.9 & 47 \\
\hline $1568 / 76$ & 8.3 & -0.3 & 0.4 & 16 & 1.9 & 1.6 & 27 \\
\hline $1373 / 76$ & 7.6 & -0.5 & 0.5 & 17 & 1.2 & 1.0 & 26 \\
\hline $3198 / 77$ & 7.6 & -0.3 & 0.5 & $2 f$ & 2.3 & 1.7 & 34 \\
\hline $1608 / 78$ & 7.3 & -0.4 & 0.4 & 21 & 1.5 & 1.2 & 20 \\
\hline$\overline{\mathrm{X}}$ & 7.9 & 0.4 & 0.5 & 18 & 1.7 & 1.2 & 28.5 \\
\hline S.D. & 0.6 & 0.08 & 0.05 & 4.5 & 0.5 & 0.3 & 0.7 \\
\hline S.E. & 0.2 & 0.04 & 0.02 & 1.6 & 0.2 & 0.1 & 3.1 \\
\hline
\end{tabular}

cult, since it was based at least in part on information obtained from the patient or his relatives.

\section{(a) Severity of the illness}

The relations between the severity of illness, the mean alpha frequency and the mean age of the patients is shown in Table II.

From this it appears that none of the EEGs was obtained from patients in stage I of the disease and most EEGs were recorded in stage

\section{TABLE II}

Relation between severity of illness (stages $I-V$ ) and mean alpha frequency. Remark: no EEGs were recorded in patients in stage I (lightest degree).

\begin{tabular}{lcllll}
\hline Stage & Number & $\begin{array}{l}\text { Mean } \\
\text { age }\end{array}$ & \multicolumn{2}{l}{ Alpha freq. } \\
\cline { 4 - 6 } & & & Mean & S.D. & S.E. \\
\hline I & - & - & - & - & - \\
II & 24 & 28 & 9.1 & 1.0 & 0.2 \\
III & 44 & 37.5 & 8.4 & 0.8 & 0.1 \\
IV & 13 & 45.5 & 7.9 & 0.8 & 0.2 \\
V & 5 & 54 & 7.5 & 0.5 & 0.3 \\
\hline
\end{tabular}

II and stage III (68). There is a clear relation between the stages and the alpha frequency.

The relation between severity of the disease and patient's age is obviously due to the chronicity of the disease and needs no further comment. It might be argued that the decrease of alpha frequency might be related to the increased patient's age, but the trend sets in between the average ages of 28 and 37.5 years, when no such considerable decrease of alpha frequency can be expected in a normal group (Lundervold et al. 1969; Wieneke et al. 1980).

\section{(b) Duration of the illness}

The duration of the illness was based on data obtained from the patient or relatives. Because of the gradual onset of the first symptoms the date at which the disease began cannot be determined precisely. The diagram in Fig. 5 demonstrates the relation between mean alpha frequency and the estimated duration of the disease at the time of the EEG recording. From this figure it appears that a decrease of mean alpha frequency is related to an increased duration of the disease. 


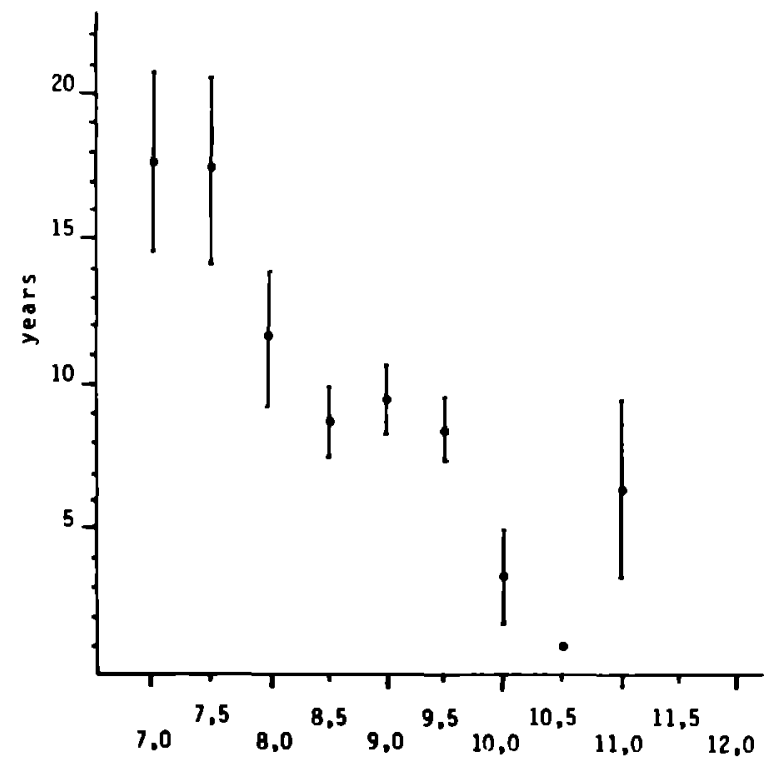

Fig. 5. Relation between duration of the disease and mean alpha frequency. On the vertical axis is indicated the number of years the patients have suffered from the disease. On the horizontal axis the mean frequency of the alpha rhythm is presented. The vertical bars represent the standard errors.

\section{Discussion}

In comparison to the investigations described in the relevant literature, our study has been carried out over a long period of time (23 years) in a rather large group of patients $(84+2)$ suffering from this rare disease. The long period of time entails that the EEG recording techniques have varied and that the most recently available analysis methods - undoubtedly of considerable value - have been performed only on a small number of EEGs $(6+2)$. Reviewing the material, it would have been advantageous if a larger number of repeated EEG records had been performed. At the time such repeated investigations were not considered to be directly in the interest of the patients themselves and it did not seem justified to ask them to travel long distances from their homes to our department. Of some of the parameters which we have investigated, e.g., the alpha amplitude and index with the eyes open, no adequate data in normal subjects were available in the literature, to our knowledge.

Taking these shortcomings into consideration it appears nevertheless allowable to make the following conclusions.

The alpha frequency in these patients is generally low, in a notable percentage $(30 \%)$ $8 \mathrm{c} / \mathrm{sec}$ or lower. The entire frequency spectrum appears to be shifted towards the low frequency side. This appears to concur not only with data described by Savoldi (1959) and Friedlander and Bittenbender (1964), but also with data recently obtained in our department in an investigation of frequency power spectra of the EEGs of 110 normal subjects in age groups evenly distributed between 17 and 50 years. In this study (Wieneke et al.) no significant differences were found between frequencies of alpha rhythms at lower and at higher ages, confirming much earlier studies by Brazier and Finesinger (1944). The mean alpha frequency of our normal subjects was $9.9 \mathrm{c} / \mathrm{sec}$ (S.D., 1.0; S.E., 0.1 ). The mean age of the patient group was 36 years and the mean alpha rhythm frequency $8.5 \mathrm{c} / \mathrm{sec}$ (S.D., 1.0; S.E., 0.1).

From these observations it can be concluded that generally in our patient population the alpha frequency is lower than in normal subjects. The fact that the alpha frequency decreases with increasing age of the patients appears to be related to the increased duration and severity of the illness rather than to ageing as such.

As far as the amplitudes of the alpha rhythms are concerned, these are comparatively small (average $44 \mu \mathrm{V}$; S.D., 15; S.E., 1.5) and do not appear to be related to the alpha frequencies. Since, however, no similar data in groups of normal subjects are available, no definite statements can be made.

The alpha indices appear to be high, with the eyes closed or open. Again, since no comparable data from normal subjects are available this statement cannot be substantiated.

The group of patients of whose EEGs computer analysis was carried out is small. Nevertheless, the data obtained in this small group 
generally concur with those obtained by manual measurements in the larger group of 84 patients. The analyses show that the frequencies are low, the bandwidths narrow (comparable with regularity of alpha rhythms) and the powers comparatively low. In addition, the bilateral and longitudinal coherences are high. Bilateral coherence indicates a considerable degree of bilateral synchrony of the alpha rhythm and longitudinal coherence indicates synchrony of the alpha rhythms in posterior and anterior areas. It may be concluded therefore that these alpha rhythms have low frequencies, are monotonous and occur over large areas while the amplitudes are nonetheless comparatively small. Since the usual spread of the alpha rhythm synchronously over large areas is related to large amplitudes, our findings might possibly indicate that in these patients a smaller number of neurones per unit area take part in the generation of alpha rhythms than is normally the case. This may be regarded as being compatible with the general degenerative nature of the disease.

\section{Summary}

An investigation has been carried out in a group of $84(+2)$ patients suffering from myotonic dystrophia (Curschmann-Steinert).

Manual measurements show that in these patients: (1) the alpha rhythms have low frequencies, related to the age of the patient and to the duration and severity of the disease; (2) the alpha rhythms are regular, monotonous; (3) the alpha rhythms have small amplitudes; there appears to be no relation between alpha frequency and amplitude; (4) the alpha indices are high during eyes closed as well as during eyes open.

The computer measurements generally appear to concur with the above and in addition show that the bilateral and longitudinal coherences are high, indicating considerable synchrony of the alpha rhythm over large areas on both sides of the head.
The possible significance of these observations is discussed.

\section{Résumé}

Observations EEG au cours de la dystrophie myotonique (Curschmann-Steinert)

On a procédé à une étude portant sur un groupe de $84(+2)$ patients souffrant de dystrophie myotonique (Curschmann-Steinert).

Des évaluations manuelles ont montré que, chez ces patients: (1) le rythme alpha a une fréquence basse, fonction de l'âge du patient, de la durée et de la gravité de la maladie; (2) il est régulier et monotone; (3) il présente une amplitude faible, et qui ne paraît pas être fonction de la fréquence; (4) les indices alpha sont élevés aussi bien les yeux ouverts que les yeux fermés.

Des traitements par ordinateur ont en général confirmé les données ci-dessus, en montrant de surcroît que les cohérences entre les dérivations bilatérales et entre les dérivations longitudinales sont élevées, preuve d'une synchronisation importante du rythme alpha sur de larges territoires des deux hémisphères.

La signification de ces résultats est discutée.

\section{References}

Barwick, D.D., Osselton, J.W. and Walton, J.N. EEG studies in hereditary myopathy. J. Neurol. Neurosurg. Psychiat., 1965, 28: 109-114.

Brazier, M.A.B. and Finesinger, J.E. Characteristics of the normal electroencephalogram. A study of the occipital cortical potentials in 500 normal subjects. J. clin. Invest., 1944, 23: 303-311.

Friedlander, W.J. and Bittenbender, J.B. EEG findings in myotonica dystrophia. Electroenceph. clin. Neurophysiol., 1964, 17: 564-566.

Jenkins, C.M. and Watts, D.G. Spectral Analysis and its Applications. Holden-Day, San Francisco, Calif., 1968,379 pp.

Lee, F.I. and Hughes, D.T.D. Systemic effects in dystrophia myotonica. Brain, 1964, 87: 521-537.

Lundervold, A. The EEG in dystrophia myotonica. 
In: 6 th Int. Congr. Electroenceph. and Neurophysiol. Vienna Commun., 1969: 293.

Lundervold, A., Refsum, S. and Jacobsen, W. The EEG in dystrophia myotonica. Europ. Neurol., 1969, 2: 279-284.

Savoldi, F. EEG pattern in Steinert's disease. Electroenceph. clin. Neurophysiol., 1959, 11: 386P.

Storm van Leeuwen, W., Spoelstra, P.J. and Wieneke, G.H. Significance of power spectra and coherence functions in routine clinical electroencephalography. In: G.K. Schenk and M. Matejeck (Eds.), Quantitative Analysis of the EEG, Vol. II. AEG Telefunken, Konstanz, 1975.

Wieneke, G.H., Deinema, C.H.A., Spoelstra, P.J., Storm van Leeuwen, W. and Versteeg, H. Some quantitative data on alpha-rhythm in 110 normal male adults. Electroenceph. clin. Neurophysiol., 1980,49 : in press. 